\title{
On the interplay between flaring and shadowing in disks around Herbig Ae/Be stars
}

\author{
B. Acke ${ }^{1, \star}$, M. $\mathrm{Min}^{2}$, M. E. van den Ancker ${ }^{3}$, J. Bouwman ${ }^{4}$, B. Ochsendorf ${ }^{2}$, A. Juhasz ${ }^{4}$, and L. B. F. M. Waters ${ }^{1,2}$ \\ ${ }^{1}$ Instituut voor Sterrenkunde, K. U. Leuven, Celestijnenlaan 200D, 3001 Leuven, Belgium \\ e-mail: bram@ster.kuleuven.be \\ 2 Sterrenkundig Instituut "Anton Pannekoek", Universiteit van Amsterdam, Kruislaan 403, 1098 SJ Amsterdam, the Netherlands \\ 3 European Southern Observatory, Karl-Schwarzschild-Strasse 2, 85748 Garching bei München, Germany \\ ${ }^{4}$ Max-Planck-Institut für Astronomie, Königstuhl 17, 69117 Heidelberg, Germany
}

Received 19 June 2009 / Accepted 8 July 2009

ABSTRACT

\begin{abstract}
Context. Based on their spectral energy distribution, Herbig stars have been categorized into two observational groups, reflecting their overall disk structure: group I members have disks with a high degree of flaring as opposed to their group II counterparts. Literature results show that the structure of the disk is a strong function of the disk mass in $\mu \mathrm{m}$-sized dust grains.

Aims. We investigate the 5-35 $\mu \mathrm{m}$ Spitzer IRS spectra of a sample of 13 group I sources and 20 group II sources. We focus on the continuum emission to study the underlying disk geometry.

Methods. We have determined the [30/13.5] and [13.5/7] continuum flux ratios. The 7- $\mu \mathrm{m}$ flux excess with respect to the stellar photosphere is measured, as a marker for the strength of the near-IR emission produced by the hot inner disk. We have compared our data to the spectra produced by self-consistent passive-disk models, for which the same quantities were derived.

Results. We confirm the results by Meijer et al. (2008, A\&A, 492, 451) that the differences in continuum emission between group I and II sources can largely be explained by a difference in amount of small dust grains. However, we report a strong correlation between the [30/13.5] and [13.5/7] flux ratios for Meeus group II sources. Moreover, the [30/13.5] flux ratio decreases with increasing 7- $\mu \mathrm{m}$ excess for all targets in the sample. To explain these correlations with the models, we need to introduce an artificial scaling factor for the inner disk height. In roughly 50\% of the Herbig Ae/Be stars in our sample, the inner disk must be inflated by a factor 2 to 3 beyond what hydrostatic calculations predict.

Conclusions. The total disk mass in small dust grains determines the degree of flaring. We conclude, however, that for any given disk mass in small dust grains, the shadowing of the outer (tens of AU) disk is determined by the scale height of the inner disk ( $\sim 1$ AU). The inner disk partially obscures the outer disk, reducing the disk surface temperature. Here, for the first time, we prove these effects observationally.
\end{abstract}

Key words. circumstellar matter - stars: pre-main sequence - planetary systems: protoplanetary disks

\section{Introduction}

Herbig Ae/Be stars are the intermediate-mass (2-8 $M_{\odot}$ ) analogues of $\mathrm{T}$ Tauri stars. They are surrounded by circumstellar disks, which are believed to be the sites of ongoing planet formation. The geometry of these disks has been the subject of numerous studies. Meeus et al. (2001) have classified the observed spectral energy distributions (SEDs) of a sample of Herbig stars into two groups: group I members display a significantly larger far-infrared excess than their group II counterparts. Dullemond \& Dominik (2004a) have provided a physical explanation for this difference: group II sources have an outer disk which is protected against direct stellar radiation by the puffed-up inner disk. However, if the outer disk emerges from the inner disk's shadow, i.e. has a large flaring angle, its SED resembles that of a group I source. Dullemond \& Dominik (2004b) and Meijer et al. (2008) indicate that the dust grain size distribution plays a determining role in the structure of these circumstellar disks and their SED appearance. This idea is supported by observational evidence: the grains responsible for the (sub-)millimeter emission are significantly smaller in group I than in group II (Acke et al. 2004).

^ Postdoctoral Fellow of the Fund for Scientific Research, Flanders.
In the early disk models, the inner disk was modeled as a vertical wall at the dust sublimation distance to the star. Later refinements (e.g., Isella \& Natta 2005) took into account the dependence of grain sublimation temperature on gas density, resulting in a curved inner rim. With the coming of age of near-IR interferometers, however, it became clear that our knowledge of the inner disk remains incomplete. For a growing list of targets (Monnier et al. 2005; Kraus et al. 2008; Acke et al. 2008; Tannirkulam et al. 2008), the inner disk appears less spatially resolved than expected from the models. This is commonly explained by the presence of an optically thick, possibly gaseous, disk component located within the dust sublimation radius. However, near-IR interferometric data have been successfully fitted with artificially increased inner disk heights as well (Verhoeff et al. 2009, submitted to A\&A).

We have collected and analyzed the Spitzer IRS (Houck et al. 2004) spectra of a sample of 54 Herbig Ae/Be stars (Bouwman, Juhasz, Acke et al. in prep). In this Letter, we focus on the shape of the underlying continuum and report the discovery of two tight correlations between the near-IR excess and mid-to-far-IR colors. We explain these correlations in the framework of selfconsistent passive-disk models (Min et al. 2009). 


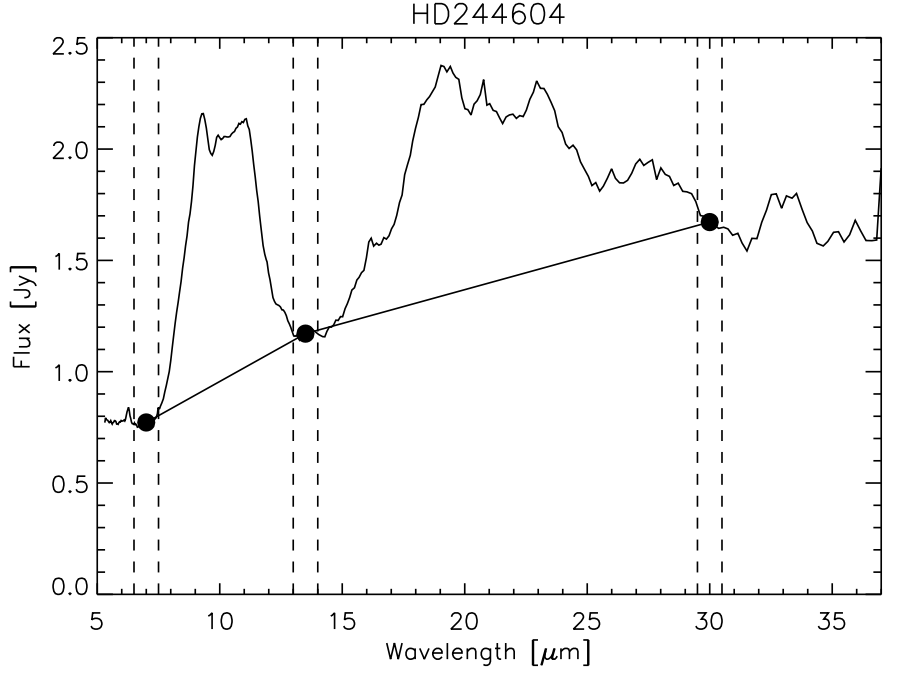

Fig. 1. Spitzer IRS spectrum of the sample target HD244604. Indicated are the continuum wavelengths at $7,13.5$ and $30 \mu \mathrm{m}$ where the spectral features of silicates and PAHs are weak or absent. The bin width is $1 \mu \mathrm{m}$.

\section{Anti-correlation between near-IR excess and far-IR color}

For all the target stars, we have fitted a reddened Kurucz (1991) model to optical photometric measurements available in the literature. From the IRAS $60 \mu \mathrm{m}$ photometry, the excess magnitude is computed $\left(M_{\mathrm{exc}}=2.5 \log \left(F_{\text {tot }} / F_{\star}\right)\right)$. The Spitzer IRS spectra are used to determine the average fluxes at 7, 13.5 and $30 \mu \mathrm{m}$ in a bin with a width of $1 \mu \mathrm{m}$. As shown in Fig. 1, only a few wavelengths are suitably free of solid-state features. The chosen bin width is a compromise between maximal signal-tonoise and minimal influence of spectral features of amorphous and crystalline silicates, and PAHs. The error bars on the measured fluxes include the errors in the individual spectral channels and the spread of the spectrum within the bin. In this paper we discuss the [13.5/7] and [30/13.5] flux ratios, and the 7- $\mu \mathrm{m}$ excess which probes the near-IR excess ${ }^{1}$. The error on the latter includes an additional $0.11 \mathrm{mag}$, as the photometric accuracy of Spitzer IRS is 10\% (Bouwman et al. 2008).

In previous work (Acke \& van den Ancker 2006), we have shown that the IRAS $60 \mu \mathrm{m}$ excess can distinguish in a simple and reliable way between the two Meeus et al. (2001) groups. Targets with a $60 \mu \mathrm{m}$ excess exceeding $10.1 \mathrm{mag}$ are classified as group I, the others as group II. Seven sources with tenuous or optically thin disks, defined as those with a total infraredto-stellar luminosity $L_{\mathrm{IR}} / L_{\star}$ below $10 \%$, are excluded as they cannot be compared to our optically thick disk models (Sect. 3). Our final sample contains 13 group I and 20 group II sources for which Spitzer IRS spectra are available covering the full range from 5 to $35 \mu \mathrm{m}$.

In Fig. 2a, the [30/13.5] flux ratio is plotted as a function of the excess at $7 \mu \mathrm{m}$. For both group I and group II sources, a clear decline in [30/13.5] flux ratio is observed with increasing 7- $\mu \mathrm{m}$ excess. As indicated by the Pearson correlation coefficient, the trend is significant with a false-positive probability (FPP) less than $1 \%$. We have performed a linear fit to the $(7-\mu \mathrm{m}$ excess, $\log [30 / 13.5])$ data of group I and II separately. The fits, indicated by the black and red line in the figure, have similar slopes: $-0.16 \pm 0.01$ and $-0.18 \pm 0.01$ for group I and II respectively.

\footnotetext{
${ }^{1}$ Approx. 70\% of the 7- $\mu \mathrm{m}$ flux is produced in the innermost disk.
}

Note, however, that the [30/13.5] flux ratios in group I are higher than those in group II for any given value of the 7- $\mu$ m excess.

Figure 2b is a plot of the [30/13.5] versus [13.5/7] flux ratio. Group II sources display a pronounced correlation between both colors (FPP $<1 \%)$. The group I sources are scattered in this diagram. A few outliers are noted in the plot. Group I sources HD34282 (\#2) and HD135344B (\#10) are the bluest sample sources in [13.5/7] color, and the reddest in [30/13.5]. These targets have indications for the presence of a large (opacity) gap in their inner disk, which explains their anomalous colors. HD37411 (\#20) and HD152404 (AK Sco, \#29) have the highest [30/13.5] ratio in group II and deviate from the trend. Also for these sources, a large disk gap may be present. The presence of a (stellar or sub-stellar) binary companion likely plays a role in opening up the gap.

\section{Comparison with disk models}

From the correlations described above, it appears that the hot inner disk $(\sim 1 \mathrm{AU})$ and the structure of the outer disk (tens of AU) closely relate to each other in the sample of Herbig stars. To understand the physics behind this result, we have computed a grid of disk models using the radiative transfer code MCMax (Min et al. 2009). The central star in these models is a mainsequence star of spectral type $\mathrm{A} 0\left(T_{\mathrm{eff}}=10000 \mathrm{~K}, R_{\star}=2 R_{\odot}\right.$, $\left.M_{\star}=2.5 M_{\odot}\right)$, representative for the median star in the sample. Altering the stellar parameters over the sample range has no influence on the analysis below.

A circumstellar disk was modeled, assuming hydrostatic equilibrium (HSE) and thermal coupling between gas and dust. The gas-to-dust mass ratio was fixed to 100 . The dust consists of astronomical silicates with a grain size of $0.12 \mu \mathrm{m}$. Large ( $>$ several $\mu \mathrm{m}$ ) grains have not been included, as they do not influence the SED shape shortward of $60 \mu \mathrm{m}$ (Meijer et al. 2008). The total dust mass in the models $(m)$ hence only refers to the total dust mass in these small grains and should be interpreted as such. We have computed models with different values for $m$. Next to the dust mass, the surface density power law $\left(\Sigma \propto R^{-p}\right)$ and the inclination of the system $(i)$ were altered. Also, we have artificially increased the inner disk's scale height by a factor $\Phi$, to study the effect of a more puffed-up inner rim than HSE calculations produce. The ranges of the model parameters are listed in Table 1. As for the sample targets, only disk models that display a $10 \mu \mathrm{m}$ silicate emission feature in their spectrum are included in the analysis. In total, 1000 disk models were included in the study. Based on their $60 \mu \mathrm{m}$ excess, roughly half would be classified as Meeus group I sources, the other half as group II.

Varying the inclination of the considered disk models only has a minor influence on the 7- $\mu \mathrm{m}$ excess (variations of $0.2 \mathrm{mag}$ ) and the IR flux ratios (25\%). A spread in inclinations therefore cannot reproduce the range, location and correlations of the observations in the diagrams. The locus of the models is predominantly determined by the mass in small dust grains (see Fig. 2c,d). The higher the mass in small dust grains, the larger the outer disk's flaring angle becomes, and hence the redder the model appears in the [30/13.5] flux ratio. However, the dust mass cannot account for the correlation that is seen between the $7-\mu \mathrm{m}$ excess and the [30/13.5] flux ratio in both groups, and the correlation between the [13.5/7] and [30/13.5] flux ratios in group II.

The power of the surface density law has a moderate influence on the location of the models in the diagrams: models with a steep decline of the surface density with radius are bluer. However, the changes induced by this parameter are insufficient to explain the observed spread in disk excess and color. To cover 


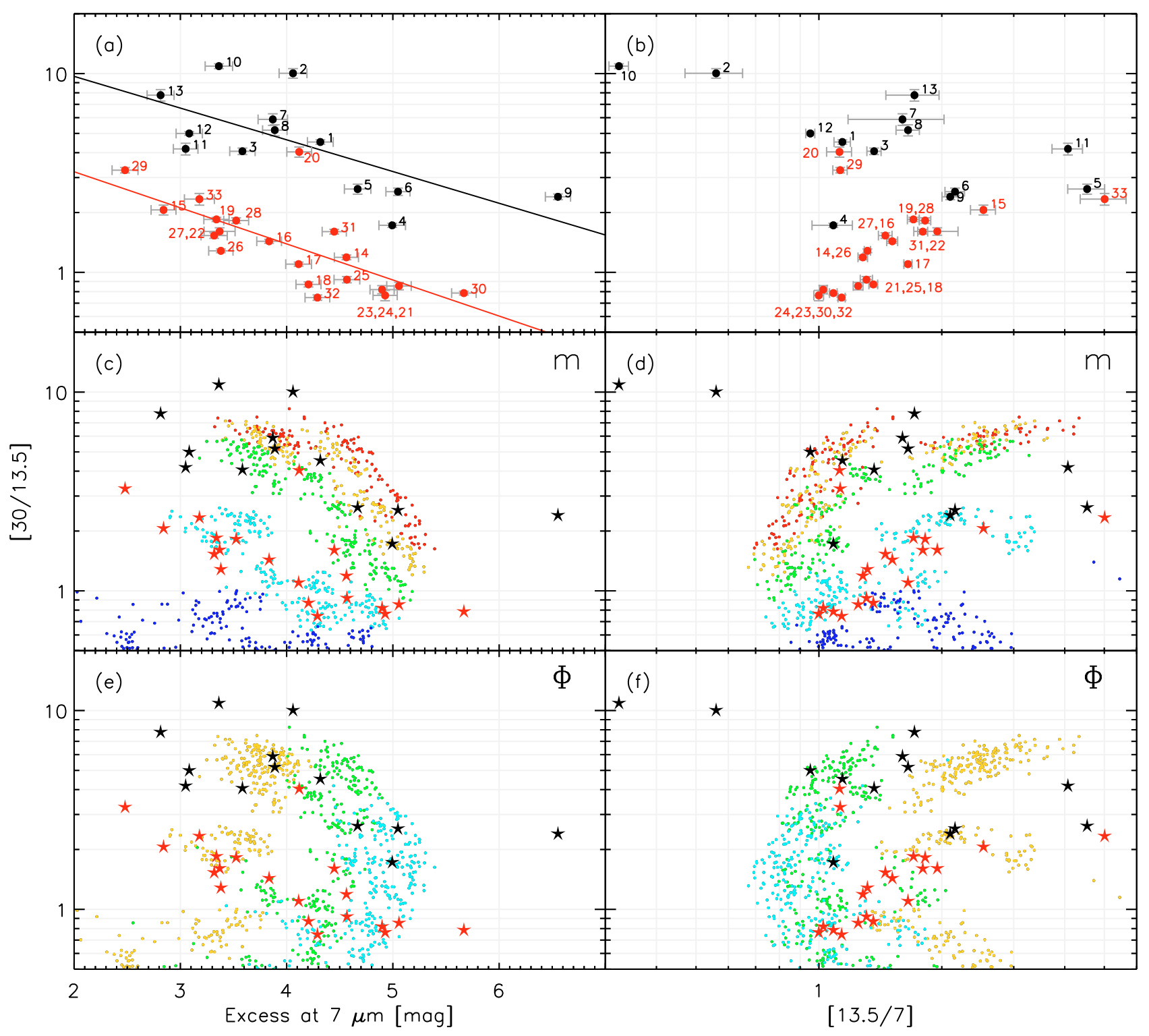

Fig. 2. Left panels: a) the clear anti-correlation between the 7- $\mu \mathrm{m}$ excess and the Spitzer [30/13.5] flux ratio. Meeus group I and II sources are resp. black and red dots. Also shown are the best linear fits to the correlation for group I (black) and II (red line). Group I: 1. HD31293, 2. HD34282, 3. HD36112, 4. RR Tau, 5. HD38120, 6. HD250550, 7. HD97048, 8. HD100453, 9. SS73 44, 10. HD135344B, 11. HD139614, 12. HD142527, 13. HD169142, Group II: 14. HD31648, 15. HD35187, 16. HD244604, 17. HD37258, 18. BF Ori, 19. HD37357, 20. HD37411, 21. HD37806, 22. HD72106S, 23. HD85567, 24. HD95881, 25. HD101412, 26. HD104237, 27. HD142666, 28. HD144432, 29. HD152404, 30. VV Ser, 31. WW Vul, 32. HD190073, 33. HD203024 c) and e): identical to panel a) with all models included (dots). The stars refer to the observations. c) The color of the dots refers to the total disk mass in small dust grains: $\log m\left[M_{\odot}\right]=-3$ to -7 from red to blue. The models shift from upper right to lower left with decreasing mass in small dust grains. e) Colors refer to the inner disk scale height parameter $\Phi$ : yellow are the models in hydrostatic equilibrium, green and blue refer to $\Phi$ equal to 2 and 3. The models shift from upper left to lower right with increasing inner rim scale height. Right panels: b) the [30/13.5] flux ratio as a function of the [13.5/7] ratio. A tight correlation between both flux ratios is noted for group II. d) and f): identical to panel b) with models included (dots). The same plotting convention as in the left panels is used. The models shift from upper left to lower right with decreasing mass in small dust grains d) and from upper right to lower left with increasing scale height f).

the full observational range, especially in 7- $\mu \mathrm{m}$ excess, we need to artificially inflate the inner disk beyond its equilibrium scale height by a factor of a few (see Fig. 2e,f).

The correlation between the [30/13.5] color and the $7-\mu \mathrm{m}$ excess displays scatter that greatly exceeds the observational errors. We take the best linear fit to the group II data (i.e. the red line in Fig. 2a) as the reference and compute the perpendicular distance of all observations to this line. This distance is strongly correlated with the magnitude of the $60 \mu \mathrm{m}$ excess: sources below the average trend have lower excesses than targets above it (see Fig. 3). This correlation with far-IR excess is significant for the sample as a whole (FPP $<1 \%$ ), but also when limited to group II sources alone (FPP $<5 \%$ ). The disk models also display the increase in $60 \mu \mathrm{m}$ excess with distance to the average fit. This confirms that the mass in small dust grains determines the locus of the targets and the disk models in the color-excess diagram, perpendicular to the observed correlation. Group I disks are more massive in small dust grains $\left(m>10^{-5} M_{\odot}\right)$, while the group II disks cluster around $m=10^{-6}-10^{-5} M_{\odot}$. Along the color-excess and color-color correlations, the defining parameter 
Table 1. Model parameter ranges.

\begin{tabular}{ll}
\hline \hline Parameter & Values \\
\hline $\log m\left[M_{\odot}\right]$ & $-3,-4,-5,-6,-7$ \\
$p$ & $1,1.5,2$ \\
$i$ & $10^{\circ}-90^{\circ}$ \\
$\Phi$ & $1,2,3$ \\
\hline
\end{tabular}

$m$ is the mass in sub- $\mu$ m-sized dust grains, $p$ is the power which governs the decline of the surface density with increasing distance to the star $\left(\Sigma \propto R^{-p}\right), i$ is the inclination of the disk with respect to the line-ofsight, $\Phi$ is the artificial scaling factor for the inner disk scale height, with $\Phi=1$ indicating hydrostatic-equilibrium models. See text for details.

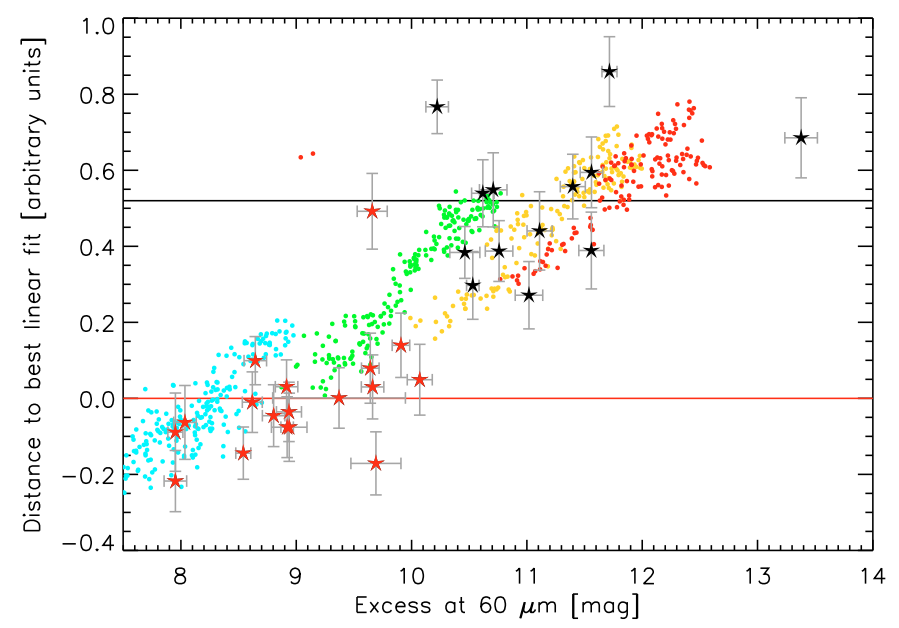

Fig. 3. The distance to the best linear fit to the group II data in Fig. 2a (red line) is strongly correlated with the $60-\mu \mathrm{m}$ excess. The black line represents the best fit to the group I data in the same figure. The stars are the group I (black) and group II data (red), the dots are the models. Colors reflect the mass in small dust grains, from $m=10^{-6}$ (bottom left) to $10^{-3} M_{\odot}$ (top right).

is the scale height of the inner disk. Roughly half of the targets can be modeled with a disk in hydrostatic equilibrium. The disks that are blue in [13.5/7] and [30/13.5] colors, however, have large $7-\mu \mathrm{m}$ excesses. For these sources, an artificially increased scale height of the inner disk is required in our models.

\section{Discussion}

The degree of flaring is the increase in opening angle of the disk surface $\alpha(r)(=\arctan (h(r) / r)$, with $h(r)$ the height of the surface above the midplane) with increasing distance to the star $r$. The disk surface is the surface that radiates in the infrared, where the opacity along the line-of-sight reaches unity. All the model disks flare, but the flaring is stronger in models with a high mass in small dust grains $\left(h(r) \propto r^{1.30}\right.$ vs. $\left.r^{1.15}\right)$. Moreover, the maximal opening angle of a massive disk is approximately 5 times that of a low-mass disk $\left(\alpha_{\max }=25^{\circ}\right.$ vs. $\left.5^{\circ}\right)$.

The observed range in $7-\mu \mathrm{m}$ excess surpasses the range predicted by hydrostatic-equilibrium models. An inflation of the inner disk height, beyond what HSE models produce, naturally leads to a higher near-IR excess, but also affects the outer disk. A disk with a puffed-up inner rim casts a large shadow over the outer disk. The surface layers of the outer disk are therefore cool and will produce hardly any infrared excess in the mid-to-farIR. The combination of both effects reduces the [30/13.5] and [13.5/7] flux ratios with increasing $7-\mu \mathrm{m}$ excess and explains the observed correlations. Note that the opening angle and degree of flaring of the disk is set by the mass in small dust grains, and only marginally depends on the height of the inner disk: $\alpha_{\max }$ decreases by $10 \%$ when $\Phi$ is increased from 1 to 3 for a given model.

\section{Conclusions}

Using Spitzer IRS spectra of a large sample of Herbig Ae/Be stars together with a large parameter set of radiative transfer models we come to the following conclusions.

- There is a strong observational correlation between the nearinfrared excess and the infrared spectral slope of Herbig $\mathrm{Ae} / \mathrm{Be}$ stars. This indicates that the apparent geometry of the outer disk is determined by that of the inner disk.

- Using radiative transfer modeling we show that the structure of the disk is governed by two parameters:

1. the mass in small dust grains which determines the degree of flaring:

2. the inner disk scale height which determines the degree of shadowing.

- Disk models in hydrostatic equilibrium fail to reproduce the observed spread in inner disk scale heights.

The findings presented in this letter together with literature infrared interferometric results, show that our understanding of the innermost regions of Herbig stars is yet incomplete. It is clear that additional physical processes, not included in our models, play a role. The correlation between the $7 \mu \mathrm{m}$ excess and the apparent geometry of the outer disk shows that the mechanism causing the flux at short wavelengths influences the outer disk. This rules out optically thin hot gas emission as an important contributor to the $7 \mu \mathrm{m}$ excess in Herbig stars. Interestingly, recent modeling efforts including gas chemistry calculations (e.g. Woitke et al. 2009) show that the gas temperatures can be much higher than the dust temperatures in the inner disk. A similar effect is obtained in disk models when including the stellar far-UV to X-ray radiation field (e.g. Gorti \& Hollenbach 2009). As a result the inner disk height is strongly increased, while the outer disk remains unaltered. Further study is needed to verify whether this affects the disk continuum emission in conformance with the observations.

Acknowledgements. This work is based on observations made with the Spitzer Space Telescope, which is operated by the Jet Propulsion Laboratory, California Institute of Technology under a contract with NASA. B.A. acknowledges support from the ESO visitor programme during the preparation of this manuscript.

\section{References}

Acke, B., \& van den Ancker, M. E. 2006, A\&A, 457, 171

Acke, B., van den Ancker, M. E., Dullemond, C. P., van Boekel, R., \& Waters, L. B. F. M. 2004, A\&A, 422, 621

Acke, B., Verhoelst, T., van den Ancker, M. E., et al. 2008, A\&A, 485, 209

Bouwman, J., Henning, T., Hillenbrand, L. A., et al. 2008, ApJ, 683, 479

Dullemond, C. P., \& Dominik, C. 2004a, A\&A, 417, 159

Dullemond, C. P., \& Dominik, C. 2004b, A\&A, 421, 1075

Gorti, U., \& Hollenbach, D. 2009, ApJ, 690, 1539

Houck, J. R., Roellig, T. L., van Cleve, J., et al. 2004, ApJS, 154, 18

Isella, A., \& Natta, A. 2005, A\&A, 438, 899

Kraus, S., Preibisch, T., \& Ohnaka, K. 2008, ApJ, 676, 490

Kurucz, R. L. 1991, BAAS, 23, 1047

Meeus, G., Waters, L. B. F. M., Bouwman, J., et al. 2001, A\&A, 365, 476

Meijer, J., Dominik, C., de Koter, A., et al. 2008, A\&A, 492, 451

Min, M., Dullemond, C. P., Dominik, C., de Koter, A., \& Hovenier, J. W. 2009, A\&A, 497, 155

Monnier, J. D., Millan-Gabet, R., Billmeier, R., et al. 2005, ApJ, 624, 832

Tannirkulam, A., Monnier, J. D., Millan-Gabet, R., et al. 2008, ApJ, 677, L51

Woitke, P., Kamp, I., \& Thi, W.-F. 2009, [arXiv:0904 .0334] 